\title{
Surgical injury: comparing open surgery and laparoscopy by markers of tissue damage
}

This article was published in the following Dove Press journal:

Therapeutics and Clinical Risk Management

\author{
Jan Máca' \\ Matúš Peteja ${ }^{2}$ \\ Petr Reimer ${ }^{\prime}$ \\ Ondřej Jor' \\ Věra Šeděnkovál \\ Lucie Panáčkovál \\ Peter Ihnát ${ }^{2}$ \\ Michal Burda ${ }^{3}$ \\ Pavel Ševčík'
}

'Department of Anesthesiology and Intensive Care Medicine, University

Hospital Ostrava, Ostrava, Czech

Republic; ${ }^{2}$ The Clinic of Surgery,

University Hospital Ostrava, Ostrava,

Czech Republic; ${ }^{3}$ Institute for

Research and Applications of Fuzzy

Modeling, Centre of Excellence

IT4Innovations, University of Ostrava,

Ostrava, Czech Republic

Correspondence: Jan Máca

Department of Anesthesiology and Intensive Care Medicine, University

Hospital Ostrava, 17, listopadu 1790,

Ostrava-Poruba 708 52, Czech Republic

Tel +420 597372713

Fax +420 597372762

Email jan.maca@fno.cz
Background: Major abdominal surgery (MAS) is high-risk intervention usually accompanied by tissue injury leading to a release of signaling danger molecules called alarmins. This study evaluates the surgical injury caused by two fundamental types of gastrointestinal surgical procedures (open surgery and laparoscopy) in relation to the inflammation elicited by alarmins.

Patients and methods: Patients undergoing MAS were divided into a mixed laparoscopy group (LPS) and an open surgery group (LPT). Serum levels of alarmins (S100A8, S100A12, HMGB1, and HSP70) and biomarkers (leukocytes, C-reactive protein [CRP], and interleukin-6 [IL-6]) were analyzed between the groups. The secondary objectives were to compare LPT and LPS cancer subgroups and to find the relationship between procedure and outcome (intensive care unit length of stay [ICU-LOS] and hospital length of stay [H-LOS]).

Results: A total of 82 patients were analyzed. No significant difference was found in alarmin levels between the mixed LPS and LPT groups. IL-6 was higher in the LPS group on day 2 $(p=0.03)$ and day $3(p=0.04)$. Significantly higher S100A8 protein levels on day $1(p=0.02)$ and day $2(p=0.01)$ and higher S100A12 protein levels on day $2(p=0.03)$ were obtained in the LPS cancer subgroup. ICU-LOS and H-LOS were longer in the LPS cancer subgroup.

Conclusion: The degree of surgical injury elicited by open MAS as reflected by alarmins is similar to that of laparoscopic procedures. Nevertheless, an early biomarker of inflammation (IL-6) was higher in the laparoscopy group, suggesting a greater inflammatory response. Moreover, the levels of S100A8 and S100A12 were higher with a longer ICU-LOS and H-LOS in the LPS cancer subgroup.

Keywords: open surgery, laparoscopy, gastrointestinal surgery, alarmins, surgical injury, major surgery

\section{Introduction}

The increasing number of major non-cardiac surgical interventions is considered a global challenge in health care. ${ }^{1}$ Extensive surgical intervention is associated with direct mechanical tissue injury, local hemorrhage, and ischemia, followed by hypoxemic cellular stress and ischemia-reperfusion injury ${ }^{2}$ leading to innate and adaptive immunity activation on the local and systemic levels. ${ }^{3}$ The dysregulation of the host response increases the number of infectious complications, impairs wound healing, and adversely affects other clinical outcomes, including hospital stay, mortality, and long-term cognitive functions. ${ }^{3-5}$ The degree of postoperative inflammatory response can be estimated by several clinical parameters (eg, heart rate, respiratory rate, and body temperature) and laboratory markers, including white blood cell (WBC) count, C-reactive protein (CRP), and interleukin-6 (IL-6) ${ }^{6-8}$ Clinical signs are unreliable and their sensitivity and specificity are low. WBC and CRP measurements are routine in clinical practice, but their kinetics are slow with low ability to differentiate sterile and 
infectious insults. ${ }^{9}$ IL-6 can be detected early, but its measurement is not widespread and thus it cannot be considered a routine biomarker. ${ }^{10}$

Alarmins are a group of intracellular molecules immediately released or actively secreted after mechanical cell injury, infection, or inflammation. In extracellular space, they exhibit cytokine-like functions, initiating an inflammatory and reparative response. ${ }^{11}$ Thus, they represent first-line signal molecules that potentially reflect the degree of tissue injury.

Laparoscopic surgical procedures (LPSs) have several advantages over open surgery, including less invasiveness (ie, smaller incision) resulting in less postsurgical pain, reduced risk of infectious contamination, and faster functional recovery. ${ }^{12}$ LPSs are the standard of care in colorectal surgery due to cost-effectiveness and better outcomes (ie, hospital stay, number of complications) compared to open surgical procedures. ${ }^{13}$ However, LPSs also have some disadvantages, such as the need for greater surgeon experience, limited range of motion, poor depth assessment, absence of tactile sensation, use of tools that are less subtle in interactions with tissues compared to the hands of a surgeon, longer duration of surgery, and general anesthesia. ${ }^{12}$

The role of alarmins in evaluating the degree of injury in relation to the type of procedure (LPS or open surgery [LPT]) in major abdominal surgery (MAS) has not yet been studied. The primary objective of the analysis was to compare the serum levels of alarmins and biomarkers between LPS and LPT in order to determine which of the procedures is more harmful. The secondary objectives were to compare alarmin and biomarker levels in cancer subgroups and find the relationship between the type of procedure and selected outcomes, specifically intensive care unit length of stay (ICU-LOS) and hospital length of stay (H-LOS).

\section{Patients and methods}

\section{Study design and patients}

The prospective single-center study was conducted between June 2013 and June 2014. The study was approved by the Medical Ethics Committee of the University Hospital of Ostrava, Czech Republic (reference number: 435/2013). The committee waived the need for informed consent prior to surgery due to the non-interventional observational nature of the study. The Consolidated Standards Of Reporting Trials flow diagram of patient selection is given in Figure 1. The inclusion criteria were as follows: $>18$ years of age, absence of sepsis at the time of admission, and elective MAS. After surgery, the patients were admitted to the intensive care unit of the Department of Anesthesiology

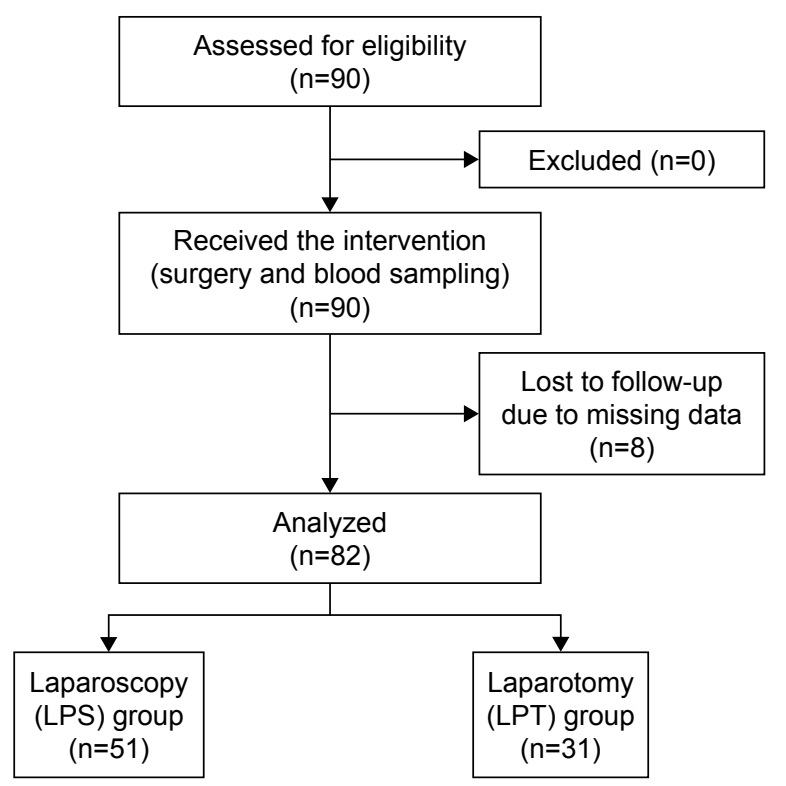

Figure I CONSORT diagram of the study selection process. Abbreviations: CONSORT, Consolidated Standards of Reporting Trials; LPS, laparoscopy; LPT, open surgery (laparotomy).

and Intensive Care Medicine, University Hospital Ostrava, Czech Republic. The exclusion criterion was pregnancy or life expectancy $<24$ hours. Major surgery was defined as a procedure expected to last $>2$ hours and/or an anticipated blood loss of $>500 \mathrm{~mL} .{ }^{14}$ General treatment, including hemodynamic optimization, use of anti-infectives, analgesia, and other measures of standard supportive care, was provided by certified intensive care physicians according to the recommendations of the Czech Society of Intensive Care Medicine ${ }^{15}$ and the Society of Critical Care Medicine. ${ }^{16}$ The whole group of patients was divided according to the type of the procedure into LPS and LPT groups. Then, in order to obtain a more homogenous cohort, we selected patients undergoing surgery for cancer and divided them into LPS cancer and LPT cancer subgroups.

\section{Study protocol and measurements}

Baseline, clinical, surgical, and outcome characteristics were obtained upon intensive care unit admission (day 0). The American Society of Anesthesiologists physical status classification before surgery was recorded. The severity of the condition was estimated using the Acute Physiology and Chronic Health Evaluation II on the first postoperative day (POD 1) and the Sequential Organ Failure Assessment on POD 1-3. The presence of systemic inflammatory response syndrome (SIRS) and sepsis at any time during the first three consecutive days after surgery was recorded according to the standard definitions valid at the time of the study. ${ }^{17}$ 
Outcome variables, such as postsurgical complications defined according to Clavien Dindo Classification, ICULOS, H-LOS, 30-day mortality, and in-hospital mortality, are given in Table 1.

Blood samples for the analysis of serum levels of biomarkers (WBC, CRP, IL-6) and alarmins (S100A8, S100A12, HMGB1, HSP70 proteins) were collected from the central venous catheter at 6:00 a.m. on POD 1-3. The blood samples were allowed to clot for $30 \mathrm{~min}$ and the supernatant subsequently centrifuged at $3,500 \mathrm{rpm}(2,500 \times g)$ for $10 \mathrm{~min}$ at $4{ }^{\circ} \mathrm{C}$. Serum concentrations of CRP (AU 5420; Beckman
Coulter, Inc., Brea, CA, USA) and IL-6 (Immulite 2000; DPC, Los Angeles, CA, USA) were assayed immediately. The interassay coefficients of variation were $4.8 \%$ and $6.7 \%$ for CRP and IL-6, respectively. Blood samples for the analysis of alarmins were centrifuged $(2,500 \times \mathrm{g})$ at $4^{\circ} \mathrm{C}$ for $6 \mathrm{~min}$ and then frozen and stored at $-80^{\circ} \mathrm{C}$ until further analyses. The concentrations of S100A8 and HMGB1 were determined quantitatively by sandwich enzyme-linked immunosorbent assay or in-house assays (Biovendor-Laboratorni medicina, Brno, Czech Republic). The concentrations of proteins S100A12 and HSP70 were determined by commercially

Table I Baseline, clinical, surgical, and outcome characteristics

\begin{tabular}{|c|c|c|c|c|}
\hline & LPT & LPS & All & $p$-value \\
\hline \multicolumn{5}{|l|}{ General characteristics } \\
\hline $\mathrm{n}$ & 31 & 51 & 82 & \\
\hline Age, years & $64(50.5-7 I)$ & $63(55.5-69.5)$ & $63(53.5-70)$ & 0.90 \\
\hline Sex, male & $21(68 \%)$ & $34(67 \%)$ & $55(67 \%)$ & 1.00 \\
\hline Body weight, kg & $82(70.5-85)$ & $80(67.5-90.5)$ & $80(68.5-89.5)$ & 0.96 \\
\hline Height, cm & $172(\mid 68-180)$ & $174(167-178)$ & $173(167.25-178.75)$ & 0.89 \\
\hline Body mass index, $\mathrm{kg} / \mathrm{m}^{2}$ & $25.95(23.62-30.11)$ & 27.44 (23.92-29.39) & $26.33(23.82-29.93)$ & 0.77 \\
\hline \multicolumn{5}{|l|}{ Clinical and surgical characteristics } \\
\hline ASA & $2(2-3)$ & $2(2-3)$ & $2(2-3)$ & 0.62 \\
\hline ASA I & $3(10 \%)$ & $3(6 \%)$ & $6(7 \%)$ & 0.67 \\
\hline ASA II & $14(45 \%)$ & $27(54 \%)$ & $4 \mid(51 \%)$ & 0.50 \\
\hline ASA III & $10(32 \%)$ & 18 (36\%) & 28 (35\%) & 0.81 \\
\hline ASA IV & $4(13 \%)$ & $2(4 \%)$ & $6(7 \%)$ & 0.20 \\
\hline APACHE II & $6.5(5-10.5)$ & $7(5-10)$ & $7(5-10.25)$ & 0.91 \\
\hline SOFA (day I) & $0(0-2.75)$ & I $(0-3.75)$ & I (0-3) & 0.42 \\
\hline SOFA (day 2) & $0(0-1.25)$ & I $(0-3)$ & I (0-3) & 0.07 \\
\hline SOFA (day 3) & $0(0-1)$ & I $(0-3)$ & I (0-3) & 0.15 \\
\hline SIRS & $29(94 \%)$ & $47(92 \%)$ & $76(93 \%)$ & 1.00 \\
\hline Sepsis & $4(13 \%)$ & $2(4 \%)$ & $6(7 \%)$ & 0.19 \\
\hline Cancer & $18(58 \%)$ & $47(92 \%)$ & $65(79 \%)$ & $<0.001$ \\
\hline Surgery, min & $220(180-265)$ & $320(240-385)$ & $275(210-360)$ & $<0.001$ \\
\hline Blood loss, mL & $300(100-525)$ & $600(175-1,050)$ & $425(100-1,000)$ & 0.04 \\
\hline Blood products (EBR), mL ${ }^{\mathrm{a}}$ & $0(0-125)$ & $0(0-550)$ & $0(0-537.5)$ & 0.35 \\
\hline Blood products (FFP), mL ${ }^{\mathrm{a}}$ & $0(0-0)$ & $0(0-555)$ & $0(0-509)$ & 0.17 \\
\hline Vasopressors & II (35\%) & $20(39 \%)$ & $31(38 \%)$ & 0.82 \\
\hline Inotropes & 7 (23\%) & $13(25 \%)$ & $20(24 \%)$ & 1.00 \\
\hline Fluids, $\mathrm{mL}$ & $2,900(2,200-4,000)$ & $4,000(2,500-4,850)$ & $3,500(2,500-4,500)$ & 0.03 \\
\hline Mechanical ventilation after surgery & $7(23 \%)$ & $13(25 \%)$ & $20(25 \%)$ & 1.00 \\
\hline Body temperature at admission, ${ }^{\circ} \mathrm{C}$ & $35.9(35.8-36.2)$ & $35.9(35.6-36.1)$ & $35.9(35.7-36.2)$ & 0.75 \\
\hline \multicolumn{5}{|l|}{ Outcome } \\
\hline Complications after surgery & $8(26 \%)$ & $23(45 \%)$ & $31(38 \%)$ & 0.45 \\
\hline \multicolumn{5}{|l|}{ Clavien Dindo Classification, yes } \\
\hline Minor & $3(10 \%)$ & II (22\%) & $14(17 \%)$ & 0.23 \\
\hline Major & $5(16 \%)$ & $12(23 \%)$ & $17(21 \%)$ & 0.58 \\
\hline ICU-LOS, days & $4(3-11.5)$ & $6(4-8)$ & $6(4-8)$ & 0.29 \\
\hline Hospital LOS, days & $15(9-24)$ & $16(11-27)$ & $16(10-25.25)$ & 0.36 \\
\hline 30-Day mortality & I (3\%) & $6(12 \%)$ & $7(9 \%)$ & 0.27 \\
\hline In-hospital mortality & $2(6 \%)$ & $7(14 \%)$ & $9(11 \%)$ & 0.47 \\
\hline
\end{tabular}

Notes: The qualitative variables are expressed as $\mathrm{n}(\%)$ and quantitative variables as the median (25th-75th percentile). ${ }^{\mathrm{a}} \mathrm{Administered}$ during surgery.

Abbreviations: ASA, American Society of Anesthesiology; APACHE, Acute Physiology and Chronic Health Evaluation; EBR, erythrocyte concentrate without buffy coat; FFP, fresh frozen plasma; ICU, intensive care unit; LOS, length of stay; LPS, laparoscopy; LPT, open surgery (laparotomy); SIRS, systemic inflammatory response syndrome; SOFA, Sequential Organ Failure Assessment. 
available colorimetric sandwich enzyme-linked immunosorbent assay kits (Biovendor-Laboratorni medicina, and StressMarq, Victoria, BC, Canada). The analyses of S100A8, S100A12, HMGB1, and HSP70 had inter-assay coefficients of variation of $5.6 \%, 6.9 \%, 10.9 \%$, and $11.3 \%$, respectively. The total WBC count was determined in a local laboratory immediately after sampling.

\section{Statistical analysis}

R software (version 3.3.1) was used for this post hoc analysis (coauthor MB) and to create the figures and tables. Numerical data are characterized as median and interquartile range. Categorical data are described as absolute and relative (\%) frequencies. To assess significant differences between groups, two-sample Wilcoxon's test or Fisher's exact test was performed (for numerical and categorical data, respectively). The level of significance was defined as 0.05 .

\section{Results}

The final statistical analysis utilized data obtained from a total of 82 patients divided into the LPT $(n=31)$ and LPS $(n=51)$ groups according to the type of surgery. Almost all patients $(93 \%, n=76)$ exhibited SIRS at some point during the first 3 days after admission. Only a small number developed sepsis $(7 \%, n=6)$. The number of complications after surgery defined according to Clavien Dindo Classification did not differ between the groups. The description of surgical interventions according to the place of resection (upper, middle, and lower gastrointestinal tract) and the type of surgery (LPS, LPT), including the number of subjects is presented in Table 2.

Baseline characteristics did not differ between the LPS and LPT groups. Comparing the clinical and surgical characteristics, we found significantly longer surgery time $(p<0.001)$, greater blood loss $(p=0.04)$, and higher amounts of intraoperatively administered fluids $(p=0.03)$ in the LPS group (sum of all laparoscopy subjects). The clinical outcomes that were followed, such as complications after surgery, ICU-LOS, H-LOS, in-hospital mortality, and 30-day mortality, did not differ between the two groups. The serum levels of alarmins (S100A8, S100A12, HMGB1, and HSP70) and biomarkers (WBC, CRP, and IL-6) in the LPS and LPT groups are given in Table 3 . We found no significant difference in serum alarmin levels between the two groups. We observed significantly higher levels of IL- 6 on day $2(p=0.03)$ and day $3(p=0.04)$ in the LPS group.

Concerning the cancer subgroups, we found no differences in baseline characteristics between LPS and LPT. However, we observed significantly longer surgery time $(p<0.001)$, greater blood loss $(p<0.001)$, higher amounts of intraoperatively administered fluids $(p<0.01)$, and higher amounts of fresh frozen plasma administered $(p=0.02)$ in the LPS cancer subgroup. In addition, ICU-LOS and H-LOS were significantly longer in the LPS cancer subgroup $(p<0.01$ for both). Number of complications, in-hospital mortality, and 30-day mortality did not differ between subgroups.

We found significantly higher levels of S100A8 protein on day $1(p=0.02)$ and day $2(p=0.01)$, and S100A12 protein on day $2(p=0.03)$ in the LPS cancer subgroup (Figure 2 ). We also observed significantly higher levels of IL-6 $(p<0.01$, for all 3 days) in the LPS cancer subgroup (Figure 3 ).

\section{Discussion}

Despite the benefits of solving the causal disease, elective major surgery causes iatrogenic trauma, eliciting a marked sterile immune response. ${ }^{3}$ The physiologic host response leads to wound healing and structural and functional restitution of a tissue. On the other hand, dysregulation of sterile inflammation can participate in the development of postoperative complications and adversely affect patient outcomes. In this study, we showed that the majority of subjects exhibited SIRS at some point during the first three consecutive days after elective MAS. This immune response to surgery and postoperative complications maybe influenced not only by injury caused by a surgeon but also by the presence of bacteria and/or bacterial products in the intestinal contents, with contamination of the surgical wound, blood,

Table 2 The description of surgical interventions according to the place of resection (upper, middle, lower GIT) and the type of surgery (LPS, LPT), including the number of subjects

\begin{tabular}{|c|c|c|c|c|c|c|}
\hline \multirow[t]{2}{*}{ GIT level } & \multirow[t]{2}{*}{ Type of surgery } & \multicolumn{2}{|l|}{ LPT } & \multicolumn{2}{|l|}{ LPS } & \multirow[t]{2}{*}{ All } \\
\hline & & Cancer & Noncancer & Cancer & Noncancer & \\
\hline Upper & Esophageal and gastric resection & 3 & 1 & 12 & I & 17 \\
\hline \multirow[t]{2}{*}{ Middle } & Pancreatic (duodenopancreatic) and hepatic resection & I & 0 & 8 & 0 & 9 \\
\hline & $\begin{array}{l}\text { Other small intestinal resections (cystectomy, status } \\
\text { ileosus, gynecologic surgery) }\end{array}$ & 0 & 8 & 10 & 0 & 18 \\
\hline Lower & Large intestine and rectum resection & 14 & 4 & 17 & 3 & $\begin{array}{l}38 \\
\mathrm{n}=82\end{array}$ \\
\hline
\end{tabular}

Abbreviations: GIT, gastrointestinal tract; LPS, laparoscopy; LPT, laparotomy. 
Table 3 Serum levels of alarmins (SI00A8, SI00AI2, HMGBI, and HSP70) and biomarkers (CRP, IL-6, albumin, and WBC) in the LPS and LPT groups three consecutive days after surgery

\begin{tabular}{|c|c|c|c|}
\hline & LPT & LPS & $p$-value \\
\hline \multicolumn{4}{|c|}{ SI00A8, ng/mL } \\
\hline Day I & I.I (0.98-I.7) & $1.24(0.93-1.68)$ & 0.50 \\
\hline Day 2 & $1.13(0.86-1.99)$ & I.45 (0.98-I.79) & 0.35 \\
\hline Day 3 & $1.04(0.83-1.62)$ & $1.21(0.77-1.84)$ & 0.68 \\
\hline \multicolumn{4}{|c|}{$\mathrm{SIOOAI} 2, \mathrm{ng} / \mathrm{mL}$} \\
\hline Day I & 89.4 (75.2-101.35) & $93(59-138.6)$ & 0.94 \\
\hline Day 2 & $96.4(86.8-116.5)$ & I $20.3(70.75-\mid 56.5)$ & 0.24 \\
\hline Day 3 & $98.05(76.88-122.75)$ & II 0.5 (7I.4-I70.I5) & 0.42 \\
\hline \multicolumn{4}{|c|}{ HSP70, ng/mL } \\
\hline Day I & $5.34(4.2 \mathrm{I}-7.33)$ & $7.64(4.82-10.29)$ & 0.06 \\
\hline Day 2 & $4.72(3.46-7)$ & $5.3(4.08-8.14)$ & 0.21 \\
\hline Day 3 & $5.13(3.42-6.46)$ & $4.68(3.16-7.13)$ & 0.99 \\
\hline \multicolumn{4}{|c|}{$\mathrm{HMGBI}, \mathrm{U} / \mathrm{mL}$} \\
\hline Day I & $14(4-46.5)$ & $12(4-58.5)$ & 0.83 \\
\hline Day 2 & $14(4-36.5)$ & II (4-50.5) & 0.90 \\
\hline Day 3 & $15.5(4-40)$ & $10(4-49.5)$ & 0.95 \\
\hline \multicolumn{4}{|c|}{ WBC count, $\times 10^{9} / \mathrm{L}$} \\
\hline Day I & $9.8(8.5-11.8)$ & $9.9(8.5-12.75)$ & 0.80 \\
\hline Day 2 & $9.4(6.65-12.65)$ & $10(8.4-12.25)$ & 0.47 \\
\hline Day 3 & $8.6(5.9-12.15)$ & $8.6(6.9-11.8)$ & 0.60 \\
\hline \multicolumn{4}{|c|}{ CRP, mg/L } \\
\hline Day I & II 5 (88-129.5) & $109(73.5-135.5)$ & 0.96 \\
\hline Day 2 & $|8|(|30.5-22|)$ & $\mid 84(|4|-2 \mid 2.5)$ & 0.82 \\
\hline Day 3 & $146(104.5-190)$ & | 45 (| |4.5-2|4.5) & 0.33 \\
\hline \multicolumn{4}{|l|}{ IL-6, ng/L } \\
\hline Day I & III (63.65-278.5) & $149(|| 0.5-28 \mid)$ & 0.13 \\
\hline Day 2 & $69.1(28.4-109.5)$ & $95.2(54.9-155.5)$ & 0.03 \\
\hline Day 3 & $31.3(16.95-48.85)$ & $45.8(23.45-93.5)$ & 0.04 \\
\hline
\end{tabular}

Note: The variables are expressed as the median (25th-75th percentile). Abbreviations: CRP, C-reactive protein; HMGBI, high mobility group box I; HSP70, heat shock protein 70; IL-6, interleukin-6; LPS, laparoscopy; LPT, open surgery (laparotomy); WBC, white blood cell.
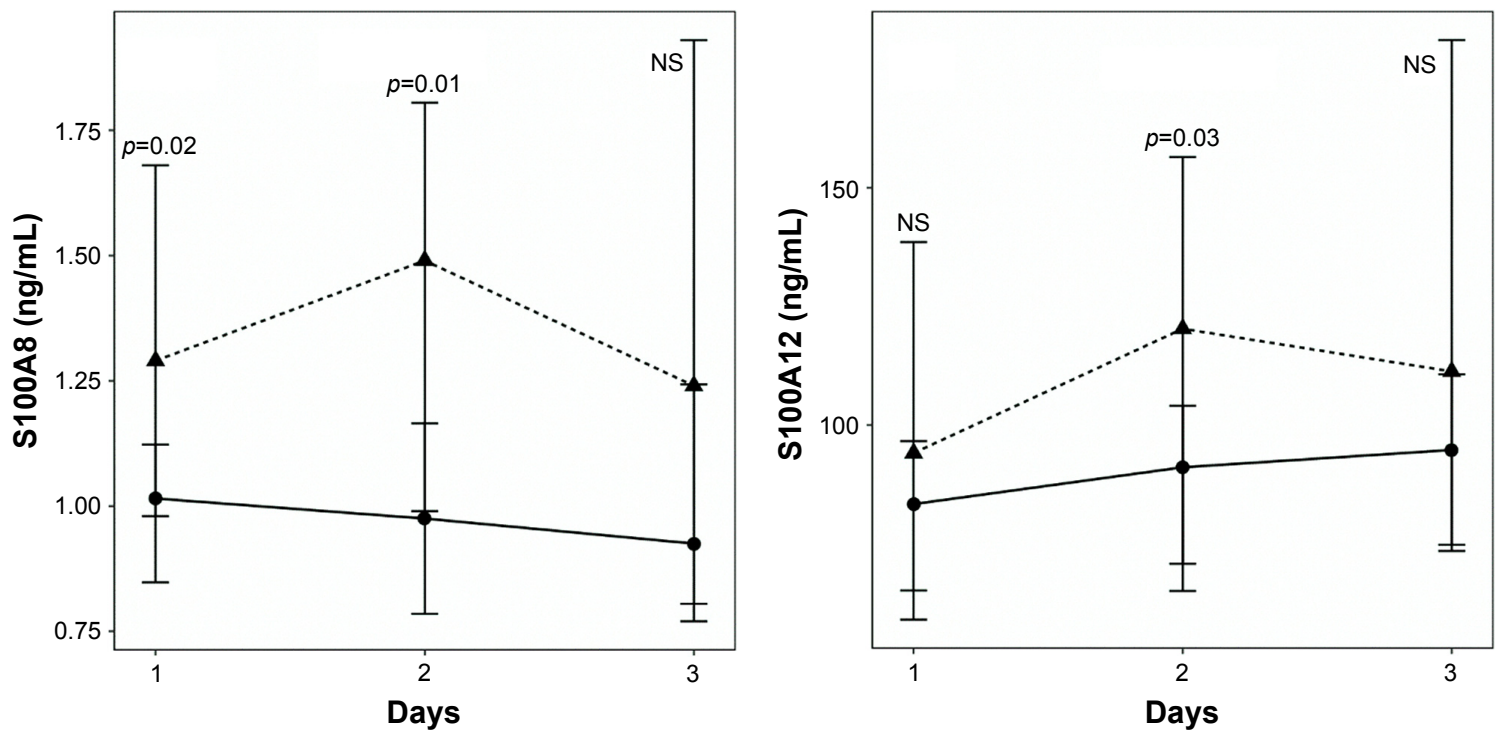

and so on. In our study, all subjects received routine antibiotic prophylaxis ( $\beta$-lactam and metronidazole). Due to the low incidence of early postoperative sepsis $(7 \%)$, we consider the anti-infective prophylaxis to be effective in decreasing the risk of infectious complications.

Our selection of the particular set of alarmins was based on their already known roles in inflammatory diseases. ${ }^{18-20}$ S100A proteins are responsible for regulating the intracellular metabolism of calcium $\left(\mathrm{Ca}^{2+}\right)$ and calcium-dependent signaling. The expression of $\mathrm{S} 100 \mathrm{~A}$ proteins is enhanced in inflamed tissues, where they activate endothelial and immune cells and facilitate the formation of local prothrombotic activity. ${ }^{21}$ HMGB1 binds to DNA and influences many nuclear processes (ie, activation of nuclear factor kappa B, repair of DNA); it is released from the injured cells and activates immunocytes. Heat shock proteins, including HSP70, bind to nascent polypeptide chains and facilitate their proper folding, working as chaperones for other danger signals and protecting cells against unfavorable conditions, primarily thermal stress. ${ }^{22}$ In preliminary data collection, we observed significantly higher levels of S100A8, S100A12, and HSP70 in surgical patients compared to healthy subjects. ${ }^{23}$ This suggests that alarmins participate in physiologic responses to surgical injury. Biomarkers, such as WBC count, CRP, IL-6, and albumin, reflect various types of injury, including infectious diseases, sterile trauma damage, and major surgery. ${ }^{8,24,25}$

Laparoscopic procedures in gastrointestinal surgery are usually preferred to open surgery, especially due to the less

- LPT cancer $\Delta$ LPS cancer

Figure 2 The significant differences between LPS and LPT cancer subgroups related to proteins SI00A8 and SI00AI2.

Abbreviations: LPS, laparoscopy; LPT, open surgery (laparotomy); NS, not significant. 


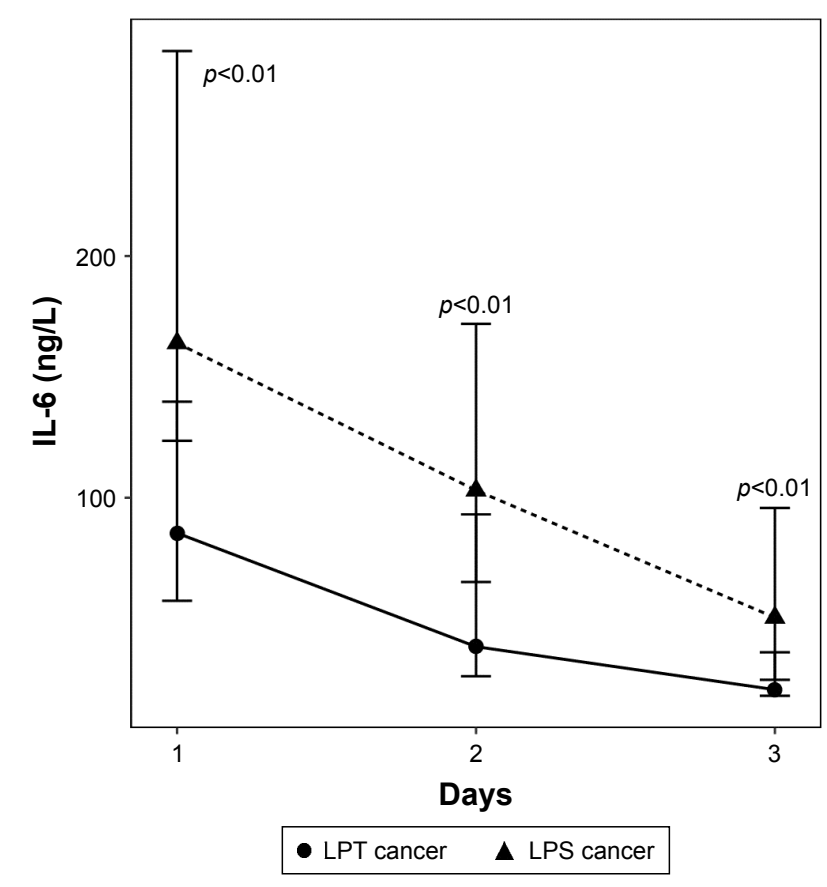

Figure 3 The significant differences between LPS and LPT cancer subgroups related to selected inflammatory biomarkers.

Abbreviations: IL-6, interleukin-6; LPS, laparoscopy; LPT, open surgery (laparotomy).

invasiveness (smaller incision), ${ }^{26}$ less postsurgical pain, reduced risk of infectious complication, faster functional recovery, and better outcomes. ${ }^{12,27}$ To date, no study has evaluated the invasiveness of surgical procedures during MAS in relation to serum alarmin levels. We found no significant difference in alarmin levels between the LPS and LPT groups, including all surgical diagnoses. Therefore, overall degree of tissue injury reflected by alarmins seems to be similar in both types of surgical procedures.

Significantly higher levels of some alarmins (S100A8, S100A12) were obtained in the LPS cancer subgroup. This confirms a higher expression of S100A proteins in cancer, ${ }^{28}$ as reflected by their more pronounced systemic release after laparoscopic surgery for cancer.

Ramanathan et al described significantly higher CRP levels in patients undergoing extensive open resection of the large intestine, compared to laparoscopic procedures. CRP had no discriminative value related to postoperative infective complications. However, the authors did not describe some of the baseline (ie, weight, body mass index) and surgical characteristics (ie, duration of surgery, blood loss, use of vasopressors). ${ }^{24}$ On the contrary, we observed no differences in CRP levels between the colorectal resection subgroups during similar postoperative periods. Moreover, we found significantly higher levels of IL-6 in the LPS cancer subgroup, suggesting a greater inflammatory host response to laparoscopy, probably reflecting the longer surgery time and higher blood loss requiring higher amount of intraoperatively administered fluids. ${ }^{29,30}$ The different findings for CRP values maybe explained by the statistical error related to fewer subjects enrolled in our study and the potentially different baseline and surgical characteristics between the studies. Fretland et al studied the influence of the type of surgery (LPS/LPT) on changes in biomarker levels after liver resection for colorectal liver metastases. The authors found higher levels of HMGB1, IL-6, CRP, cell-free DNA, and macrophage inflammatory protein $1 \beta$ in the open surgery group. ${ }^{31}$ This is again only in partial agreement with our study, because we found no difference in HMGB1 and CRP levels. These results may be explained by differences in organ-specific resections. However, we observed a significant difference in IL-6 laparoscopy group, suggesting a greater inflammatory response despite the levels of selected alarmins being similar. We suggest that a different set of alarmins other than those presently selected might be responsible for the observed inflammatory response.

We found significantly reduced ICU-LOS and H-LOS in the LPT cancer subgroup. These findings partially contradict the results reported by Zhuang et al, who described reduced H-LOS in cancer patients who underwent LPSs ${ }^{32}$ even in the setting of suboptimal use of the enhanced recovery after surgery protocol. Interestingly, we did not observe any significant differences in ICU-LOS and H-LOS between the LPS and LPT groups for all subjects undergoing MAS. We assume that this was due to the heterogeneity of the intervention used in different gastrointestinal sites and variability in the type and extensiveness of surgical diagnosis.

This study has several limitations. The first limitation is that it was designed as a post hoc analysis. The second limitation is the small numbers of subjects. Third, the intervals at which we sampled blood may not reflect exactly the dynamics of the blood concentration of alarmins. Fourth, a different set of alarmins may be responsible for the observed immune response, such as mitochondrial alarmins (mtDNA, $\mathrm{N}$-formyl-Met-Leu-Phe); thus, the physiologic role of our selected set of alarmins in the development of inflammatory response could only be minor. Fifth, the various degrees and types of cancer as well as the position of gastrointestinal tract resection are potential sources of further bias.

\section{Conclusion}

Both open surgery and laparoscopy can cause significant damage to the tissues. This study compares the degree of 
injury caused by these two main types of surgical procedures, as reflected by alarmins (danger signals and markers of tissue damage) and selected inflammatory biomarkers.

The degree of surgical injury elicited by open MAS as reflected by alarmins was similar to that of laparoscopic procedures. However, the laparoscopic surgery, especially for gastrointestinal cancer, seems to be injurious than open procedures, reflecting the longer surgery time and higher blood loss of the procedures. This is suggested by not only higher levels of biomarkers (IL-6) but also alarmins (S100A8 and S100A12), the markers of cellular damage more accurately estimating the degree of surgical injury.

\section{Acknowledgments}

Michal Burda was supported by the NPU II project LQ1602 "IT4Innovations excellence in science" provided by the MŠMT. The corresponding author would like to acknowledge Dr Zdenek Svagera, Ph.D., Head of Biochemistry, University Hospital of Ostrava, Czech Republic, and colleagues Vera Sedenkova, M.D. and Lucie Panackova, M.D. for their invaluable support to the study.

\section{Disclosure}

The authors report no conflicts of interest in this work.

\section{References}

1. Jakobson T, Karjagin J, Vipp L, et al. Postoperative complications and mortality after major gastrointestinal surgery. Medicina (Kaunas). 2014;50(2):111-117.

2. Weigand K, Brost S, Steinebrunner N, Büchler M, Schemmer P, Müller M. Ischemia/reperfusion injury in liver surgery and transplantation: pathophysiology. HPB Surg. 2012;2012:176723.

3. Marik PE, Flemmer M. The immune response to surgery and trauma: implications for treatment. J Trauma Acute Care Surg. 2012;73(4): 801-808.

4. NeSmith EG, Weinrich SP, Andrews JO, Medeiros RS, Hawkins ML, Weinrich M. Systemic inflammatory response syndrome score and race as predictors of length of stay in the intensive care unit. Am J Crit Care. 2009;18(4):339-346.

5. Eriksson LI, Kramer JH, Leung JM, et al. Perioperative cognitive decline in the aging population. Mayo Clin Proc. 2011;86(9):885-893.

6. Lamm G, Auer J, Weber T, Berent R, Ng C, Eber B. Postoperative white blood cell count predicts atrial fibrillation after cardiac surgery. J Cardiothorac Vasc Anesth. 2006;20(1):51-56.

7. Nuremberg GH, Care I, Nuremberg GH, et al. C-reactive protein and the acute phase reaction in geriatric patients. Z Gerontol Geriatr. 2015;48(7):595-600.

8. Okeny PK, Ongom P, Kituuka O. Serum interleukin-6 level as an early marker of injury severity in trauma patients in an urban low-income setting: a cross-sectional study. BMC Emerg Med. 2015;15:1-7.

9. Jain S, Gautam V, Naseem S. Acute-phase proteins: as diagnostic tool. J Pharm Bioallied Sci. 2011;3(1):118-127.

10. Tanaka T, Narazaki M, Kishimoto T. IL-6 in inflammation, immunity, and disease. Cold Spring Harb Perspect Biol. 2014;6(10):a016295.

11. Bianchi ME. DAMPs, PAMPs and alarmins: all we need to know about danger. J Leukoc Biol. 2007;81(1):1-5.
12. Joann Somers, MD. High Quality Obstetrical \& Gynecological Care, 2011-2015 [webpage on the Internet]. Available from: http://www.joannsomersmd.com/health/surgeries.php. Accessed December 27, 2017.

13. Huang C, Shen J, Zhang J, et al. Clinical comparison of laparoscopy vs open surgery in a radical operation for rectal cancer: a retrospective case-control study. World J Gastroenterol. 2015;21:13532-13541.

14. Davies SJ, Francis J, Dilley J, et al. Measuring outcomes after major abdominal surgery during hospitalization: reliability and validity of the Postoperative Morbidity Survey. Perioper Med (London). 2013;2(1):1.

15. Czech Society of Intensive Care Medicine guidelines, 2014 [webpage on the Internet]. Available from: http://www.csim.cz/dokumenty/\#cat egories:path=doporucene-postupy. Accessed December 10, 2017.

16. Society of Critical Care Medicine. Guidelines, 2016 [webpage on the Internet]. Available from: http:/www.sccm.org/Research/Guidelines/ Pages/Guidelines.aspx. Accessed December 10, 2017

17. Bone R, Balk R, Cerra F, et al. Definitions for sepsis and organ failure and guidelines for the use of innovative therapies in sepsis. The ACCP/ SCCM Consensus Conference Committee. American College of Chest Physicians/Society of Critical Care Medicine. Chest. 1992;101(6): 1644-1655.

18. Foell D, Wittkowski H, Vogl T, Roth J. S100 proteins expressed in phagocytes: a novel group of damage-associated molecular pattern molecules. J Leukoc Biol. 2007;81(1):28-37.

19. Lotze M, Tracey K. High-mobility group box 1 protein (HMGB1): nuclear weapon in the immune arsenal. Nat Rev Immunol. 2005;5(4): $331-342$.

20. Hartl F, Hayer-Hartl M. Molecular chaperones in the cytosol: from nascent chain to folded protein. Science. 2002;295(5561):1852-1858.

21. Heizmann CW, Fritz G, Schäfer BW. S100 proteins: structure, functions and pathology. Front Biosci. 2002; 7:d1356-d1368.

22. Tsan M-F, Gao B. Heat shock proteins and immune system. J Leukoc Biol. 2009;85(6):905-910.

23. Maca J, Holub M, Bursa F, et al. Alarmins as biomarkers of gastrointestinal surgical injury - a pilot study. APMIS. 2018;126(2): $152-159$.

24. Ramanathan ML, MacKay G, Platt J, Horgan PG, McMillan DC. The impact of open versus laparoscopic resection for colon cancer on C-reactive protein concentrations as a predictor of postoperative infective complications. Ann Surg Oncol. 2015;22(3):938-943.

25. Deirmengian GK, Zmistowski B, Jacovides C, O’Neil J, Parvizi J. Leukocytosis is common after total hip and knee arthroplasty. Clin Orthop Relat Res. 2011;469(11):3031-3036.

26. Conzo G, Tartaglia E, Gambardella C, et al. Minimally invasive approach for adrenal lesions: systematic review of laparoscopic versus retroperitoneoscopic adrenalectomy and assessment of risk factors for complications. Int J Surg. 2016;28(Suppl 1):S118-S123.

27. Conzo G, Pasquali D, Gambardella C, et al. Long-term outcomes of laparoscopic adrenalectomy for Cushing disease. Int J Surg. 2014; 12(Suppl 1):S107-S111.

28. Chen H, Xu C, Jin Q, Liu Z. S100 protein family in human cancer. Am J Cancer Res. 2014;4(2):89-115.

29. Barbaros U, Asoglu O, Seven R, et al. The comparison of laparoscopic and open ventral hernia repairs: a prospective randomized study. Hernia. 2007;11(11):51-56.

30. Castro PM, Rabelato JT, Monteiro GG, del Guerra GC, Mazzurana M, Alvarez GA. Laparoscopy versus laparotomy in the repair of ventral hernias: systematic review and meta-analysis. Arg Gastroenterol. 2014;51(3):205-211.

31. Fretland A, Sokolov A, Postriganova N, et al. Inflammatory response after laparoscopic versus open resection of colorectal liver metastases. Data from the Oslo-CoMet Trial. Med (Baltimore). 2015;94(42):e1786.

32. Zhuang CL, Huang DD, Chen FF, et al. Laparoscopic versus open colorectal surgery within enhanced recovery after surgery programs: a systematic review and meta-analysis of randomized controlled trials. Surg Endosc. 2015;29(9):2091-2100. 


\section{Publish your work in this journal}

Therapeutics and Clinical Risk Management is an international, peerreviewed journal of clinical therapeutics and risk management, focusing on concise rapid reporting of clinical studies in all therapeutic areas, outcomes, safety, and programs for the effective, safe, and sustained use of medicines. This journal is indexed on PubMed Central, CAS,

EMBase, Scopus and the Elsevier Bibliographic databases. The manuscript management system is completely online and includes a very quick and fair peer-review system, which is all easy to use. Visit http://www.dovepress.com/testimonials.php to read real quotes from published authors.

Submit your manuscript here: http://www.dovepress.com/therapeutics-and-clinical-risk-management-journal 